\title{
HYPERBOLIC FLOWS ARE TOPOLOGICALLY STABLE
}

\author{
Sung Kyu Choi and Jong Suh Park
}

We show that any hyperbolic flow $(X, \pi)$ on a metric space $X$ is topologically stable by showing that it is expansive and has the chain-tracing property.

\section{INTRODUCTION}

In this paper we show that the following theorem:

Theorem A. Any hyperbolic flow $(X, \pi)$ on a metric space $X$ is topologically stable.

This is an attempt to approach some problems of smooth dynamical systems theory from a non-differential point of view.

Let $(X, \pi)$ be a flow on a connected metric space $(X, d)$. For brevity we denote $x t=\pi(x, t)$ for all $x \in X$ and $t \in R$. For a point $x$ in $X$ and a number $a>0$, we define subsets of $X$ :

and

$$
\begin{aligned}
& W^{+}(x, a)=\left\{y \in X: d(x t, y t) \leqslant a \text { for all } t \in \mathbf{R}^{+}\right\} \\
& W^{-}(x, a)=\left\{y \in X: d(x t, y t) \leqslant a \text { for all } t \in \mathbf{R}^{-}\right\}
\end{aligned}
$$

A flow $(X, \pi)$ is called hyperbolic if there are positive constants $a_{0}, b_{0}, c, r$ such that

(i) $W^{+}\left(x, a_{0}\right)=\left\{y \in X: d(x t, y t) \leqslant c e^{-r t} d(x, y)\right.$ for all $\left.t \in R^{+}\right\}$, $W^{-}\left(x, a_{0}\right)=\left\{y \in X: d(x t, y t) \leqslant c e^{r t} d(x, y)\right.$ for all $\left.t \in \mathbf{R}^{-}\right\}$;

(ii) for any $(x, y) \in D\left(b_{0}\right)=\left\{(x, y) \in X \times X: d(x, y)<b_{0}\right\}$, there exists a unique element $\langle x, y\rangle \in X$ such that

$$
W^{+}\left(x v(x, y), a_{0}\right) \cap W^{-}\left(y, a_{0}\right)=\{\langle x, y\rangle\}
$$

where $v: D\left(b_{0}\right) \rightarrow \mathbf{R}$ and $\langle\rangle:, D\left(b_{0}\right) \rightarrow X$ are continuous maps.

Received 3 April 1990

The present studies were supported by the Basic Science Research Institute Program, Ministry of Education, Korea, 1989.

Copyright Clearance Centre, Inc. Serial-fee code: 0004-9729/91 \$A2.00+0.00. 
A flow $(X, \pi)$ is said to be expansive if for any constant $a>0$ there exists a constant $b>0$ with the property that if for all $t \in R, d(x t, y f(t))<b$ for a pair of points $x, y \in X$ and a continuous map $f: \mathbf{R} \rightarrow \mathbf{R}$ with $f(0)=0$, then $y=x t$, where $|t| \leqslant a$.

Let $a$ and $p$ be positive numbers. A sequence $\left(x_{i}, t_{i}\right)_{i=-m}^{n}, 0 \leqslant m, n<\infty$, in $X \times \mathbf{R}$ is called an $(a, p)$-chain if $t_{i} \geqslant p,-m \leqslant i \leqslant n$ and $d\left(x_{i} t_{i}, x_{i+1}\right)<a$, $-m \leqslant i \leqslant n$.

Let $\left(x_{i}, t_{i}\right)_{i=-m}^{n}$ be an $(a, p)$-chain in $X \times R$. We assume that

$$
T(j, k)= \begin{cases}\sum_{i=j}^{k} t_{i} & \text { if } j<k \\ 0 & \text { if } j>k\end{cases}
$$

as notation. For a point $x_{0} \in X$ and $t$ with $-T(-m,-1) \leqslant t \leqslant T(0, n)$, we define

$$
x_{0} * t= \begin{cases}x_{-j}(t+T(-j,-1)) & \text { if }-T(-j,-1) \leqslant t<-T(-j+1,-1), \\ x_{k}(t-T(0, k-1)) & \text { if } T(0, k-1) \leqslant t<T(0, k), \\ x_{n} t_{n} & \text { if } t=T(0, n) .\end{cases}
$$

For a number $b>0$, an $(a, p)$-chain $\left(x_{i}, t_{i}\right)_{i=-m}^{n}$ in $X \times \mathbf{R}$ is $b$-traced if there exists a monotone increasing continuous map $f:[-T(-m,-1), T(0, n)] \rightarrow \mathrm{R}$ satisfying

(i) $f(0)=0$,

(ii) $d\left(x f(t), x_{0} * t\right)<b$ for all $t \in[-T(-m,-1), T(0, n)]$.

A flow $(X, \pi)$ has a chain tracing property with respect to $p>0$ if for any $b>0$ there is an $a>0$ such that every $(a, p)$-chain is $b$-traced by some point in $X .(X, \pi)$ has a chain tracing property if it has a chain tracing property with respect to every positive number. If $(X, \pi)$ has a chain tracing property with respect to time 1 , then it has a chain tracing property [5].

A flow $(X, \pi)$ is called topologically stable if for any $a>0$ there exists a $b>0$ such that for every other flow $\left(X, \pi^{\prime}\right)$ with $d\left(\pi_{t}, \pi_{t^{\prime}}\right)=\sup _{x \in X} d\left(\pi_{t}(x), \pi_{t^{\prime}}(x)\right)$, where $\pi_{t}(x)=\pi(t, x)$, for all $t \in[0,1]$, then there exists a continuous map $h: X \rightarrow X$ such that $d\left(h\right.$, id) $<a$ and $h$ (orbit of $\left.\pi^{\prime}\right) \subseteq$ (orbit of $\pi^{\prime}$ ), where id is the identity homeomorphism.

Now, we list well-known results ([1] and [5]).

Theorem B. (Bowen and Walters). If a flow $(X, \pi)$ is expansive, then all fixed points are isolated.

THEOREM C. (Thomas). Every continuous expansive flow without fixed points which has the chain tracing property is topologically stable.

Then Theorem A follows from the following theorems. 
THEOREM D. Any hyperbolic flow is expansive.

THEOREM E. Any hyperbolic flow has the chain tracing property.

Thus it suffices to prove Theorems D and E, and we need some lemmas in the next section to prove these theorems.

For any homeomorphism on a compact metric space, Ombach [3] showed further relations: pseudo-orbit tracing property, expansiveness and hyperbolicity.

Basic terminologies are followed from [4].

\section{TWO LEMMAS}

Lemma 1. Let $(X, \pi)$ be a hyperbolic flow. If, for any $a<a_{0}$, there exists a number $b>0$ with $d(x, y)<b$, then

(i) $|v(x, y)| \leqslant a$,

(ii) $W^{+}(x v(x, y), a) \cap W^{-}(y, a)=\{(x, y)\}$.

Proof: Since $v(x, x)=0$ and $\langle x, x\rangle=x$, there is a number $b<b_{0}$ such that $d(x, y)<b$ implies $|v(x, y)| \leqslant a$ and

and

$$
\begin{gathered}
d(x,\langle x, y\rangle) \leqslant a / 2 c, d(y,\langle x, y\rangle) \leqslant a / c \\
d(x, x v(x, y)) \leqslant a / 2 c
\end{gathered}
$$

by the uniform continuity. Since $\langle x, y\rangle \in W^{+}\left(x v(x, y), a_{0}\right) \cap W^{-}\left(y, a_{0}\right)$, we have

$$
\begin{gathered}
d(x(v(x, y)+t),\langle x, y\rangle t) \leqslant c e^{-r t} d(x v(x, y),\langle x, y\rangle) \leqslant a, \\
d(y(-t),\langle x, y\rangle(-t)) \leqslant c e^{-r t} d(y,\langle x, y\rangle) \leqslant a
\end{gathered}
$$

for all $t \in R$. Thus

$$
\langle x, y\rangle \in W^{+}(x v(x, y), a) \cap W^{-}(y, a) \subset W^{+}\left(x v(x, y), a_{0}\right) \cap W^{-}\left(y, a_{0}\right) .
$$

Another important property of hyperbolic flows is the following.

Lemma 2. Let $(X, \pi)$ be a hyperbolic flow and $a>0$ be a constant. Suppose that there exists a constant $b>0$ such that for all $t \in \mathbf{R}$,

$$
d(x t, y(t+f(t))) \leqslant b,
$$

where $f: \mathbf{R} \rightarrow \mathbf{R}$ is a continuous map with $f(0)=0$. Then we have

(i) $|v(x, y)| \leqslant a$,

(ii) $y=x v(x, y)$. 
Proof: We can choose $a<\min \left\{a_{0} / 8, a_{0} / 2 c\right\}$ and

$$
\max \{d(x, x t): x \in X,|t| \leqslant 4 a\} \leqslant a_{0} / 8 .
$$

By Lemma 1 , there is a constant $b>0$ such that $d(x, y) \leqslant b$ implies

and

$$
|v(x, y)| \leqslant a
$$

$$
W^{+}(x v(x, y), a) \cap W^{-}(y, a)=\{\langle x, y\rangle\} .
$$

Let $v=v(x, y)$ and $z=\langle x, y\rangle$. Clearly $d(x, y) \leqslant b$ since $f(0)=0$. Put

and

$$
\begin{aligned}
& U=\left\{t \in \mathbf{R}^{+}:|f(t)| \geqslant 3 a \text { or } d(y t, z t) \geqslant a_{0} / 2\right\} \\
& V=\left\{t \in \mathbf{R}^{-}:|f(t)| \geqslant 3 a \text { or } d(x(v+t), z t) \geqslant a_{0} / 2\right\} .
\end{aligned}
$$

There exists an $s=\min U$ if $U \neq \emptyset$. Moreover, $0 \notin U$ since $|f(0)|<3 a$ and $d(y, z)<a_{0} / 2$. It follows that $s>0$.

We claim that $d(y(s-t), z(s-t)) \leqslant a_{0} / 2$ for all $t \in \mathbf{R}^{+}$. If $0<t \leqslant s$, then $d(y(s-t), z(s-t))<a_{0} / 2$ since $0 \leqslant s-t<s$ and so $s-t \notin U$. Thus we have $d(y s, z s) \leqslant a_{0} / 2$ if $t \rightarrow 0$. If $s<t$, then

$$
d(y(s-t), z(s-t)) \leqslant c e^{r(\lrcorner-t)} d(y, z)<a_{0} / 2 .
$$

It is clear that $|f(s)| \leqslant 4 a$. For all $t \in \mathbf{R}^{+}$, we have

$$
\begin{aligned}
d(y(s+ & f(s)-t), z(s+f(s)-t)) \\
\leqslant & d(y(s+f(s)-t), y(s-t))+d(y(s-t), z(s-t)) \\
& +d(z(s-t), z(s+f(s)-t)) \\
< & a_{0} .
\end{aligned}
$$

This means that $z(s+f(s)) \in W^{-}\left(y(s+f(s)), a_{0}\right)$. Also, $z(s+f(s)) \in$ $W^{+}\left(x(s+f(s)+v), a_{0}\right)$ because

$$
\begin{aligned}
d(x(s+ & f(s)+v+t), z(s+f(s)+t)) \\
\leqslant & d(x(s+f(s)+v+t), x(s+v+t))+d(x(s+v+t), z(s+t)) \\
& +d(z(s+t), z(s+f(s)+t)) \\
< & a_{0}
\end{aligned}
$$

for all $t \in \mathbf{R}^{+}$. Since $|f(s)+v| \leqslant|f(s)|+|v| \leqslant 4 a$ and $d(x s, y(s+f(s))) \leqslant b$, we have $|v(x s, y(s+f(s)))|=|f(s)+v| \leqslant a$ and $\langle x s, y(s+f(s))\rangle=z(s+f(s))$. Furthermore, we have

$$
\begin{aligned}
d(y s, z s) \leqslant & d(y s, y(s+f(s)))+d(y(s+f(s)), z(s+f(s))) \\
& +d(z(s+f(s)), z s) \\
& <a_{0} / 2
\end{aligned}
$$


since $d(y(s+f(s)), z(s+f(s))) \leqslant a$ and $|f(s)|<|f(s)+v|+|v| \leqslant 2 a$. This contradicts the fact that $s \in U$. Hence $U=\emptyset$. Also, we obtain $V=\emptyset$ by a similar method.

Now, let $A>0$ be any number and $t \in \mathbf{R}^{-}$. When $t \geqslant-A$,

and

$$
d(y(A+t), z(A+t)) \leqslant a_{0} / 2
$$

$$
d(y(A+t), z(A+t)) \leqslant c e^{r(A+t)} d(y, z)<a_{0} / 2
$$

when $t \leqslant-A$. Therefore $z A \in W^{-}\left(y A, a_{0} / 2\right)$. It follows that

$$
d(y, z)=d((y A)(-A),(z A)(-A)) \leqslant c e^{-r A} d(y A, z A) \leqslant c a_{0} e^{-r A} / 2 .
$$

For any $t \in \mathbf{R}^{+}$, we have

$$
d(x(v-A+t), z(-A+t)) \leqslant a_{0} / 2
$$

when $t \leqslant A$ and

$$
d(x(v-A+t), z(-A+t)) \leqslant c e^{r(A-t)} d(x v, z) \leqslant a_{0} / 2
$$

when $t \geqslant A$. Thus $z(-A) \in W^{+}\left(x(v-A), a_{0} / 2\right)$. This implies that

$$
\begin{aligned}
d(x z, v) & =d(x(v-A),(z(-A)) A) \leqslant c e^{-r A} d(x(v-A), z(-A)) \\
& \leqslant c a_{0} e^{-r A} / 2 .
\end{aligned}
$$

Consequently, we have

$$
d(x v, y) \leqslant d(x v, z)+d(z, y) \leqslant c a_{0} e^{-r A}
$$

and hence $d(x v, y)=0$ when $A \rightarrow \infty$. This completes the proof.

\section{Two Theorems}

Theorem D. Any hyperbolic flow $(X, \pi)$ is expansive.

Proof: For any $a>0$, we can choose a number $b>0$ by Lemma 2. We define $g: \mathbf{R} \rightarrow \mathbf{R}$ by $g(t)=f(t)-t$. Then we have $g(0)=0$ and

$$
d(x t, y(t+g(t)))=d(x t, y f(t))<b .
$$

Also, by Lemma 2, we have $|v(x, y)| \leqslant a$ and $y=x v(x, y)$. This means that $(X, \pi)$ is expansive. 
THEOREM E. Any hyperbolic flow $(X, \pi)$ has the chain tracing property.

Proof: For any $a>0$, there is a $p>0$ such that $d(x, x t)<a / 3$ for all $|t| \leqslant p$ and $x \in X$. Also, there is a $q_{1}>0$ such that $d(x f(t), y g(t))<q_{1}$ for all $A \leqslant t \leqslant B$, $A<0<B$ and continuous maps $f, g: \mathbf{R} \rightarrow \mathbf{R}$ with $f(0)=0=g(0)$, implying $|f(t)-g(t)|<p / 2$.

Putting $q=\min \left\{q_{1} / 2, a / 3\right\}$ there is a $b>0$ such that for any $(b, 1)$-chain $\left(z_{i}, s_{i}\right)_{i=-m}^{n}, 0 \leqslant m, n \leqslant \infty$ in $X \times \mathbf{R}$, there are a monotone increasing continuous map $g:[-S(-m,-1), S(0, n)] \rightarrow \mathbf{R}$ and a point $x \in X$ such that $g(0)=0$,

$$
3 t / 4-S(-m,-1) / 2-1<g(t)<5 t / 4+S(-m,-1) / 2+1,
$$

and

$$
d\left(z g(t), z_{0} * t\right)<q
$$

for all $t \in[-S(-m,-1), S(0, n)]$.

Now, let $\left(x_{i}, t_{i}\right)_{i=-\infty}^{\infty}$ be a $(b, 1)$-chain and $n_{1}=1$. We can choose $n_{k+1}>n_{k}$ so that

$$
T\left(0, n_{k+1}\right)>5 T\left(0, n_{k}\right) / 3+2 / 3 .
$$

Since $\left(x_{i}, t_{i}\right)_{i=-n_{k}}^{n_{k}}$ is also a $(b, 1)$-chain, there are $y_{k} \in X$ and a monotone increasing continuous function $g_{k}:\left[a_{k}, b_{k}\right] \rightarrow \mathrm{R}$ such that

$$
3 t / 4+a_{k} / 2-1<g_{k}(t)<5 t / 4-a_{k} / 2+1,
$$

where $a_{k}=-T\left(-n_{k},-1\right)$ and $b_{k}=T\left(0, n_{k}\right)$, and

$$
g\left(y_{k} g_{k}(t), x_{0} * t\right)<q
$$

We may assume that $y_{k} \rightarrow x$ as $k \rightarrow \infty$. Since

$$
d\left(y_{k} g_{k}(t), y_{k+1} g_{k+1}(t)\right) \leqslant d\left(y_{k} g_{k}(t), x_{0} * t\right)+d\left(x_{0} * t, y_{k+1} g_{k+1}(t)\right)<2 q
$$

for all $t \in\left[a_{k}, b_{k}\right] \subset\left[a_{k+1}, b_{k+1}\right]$, we have $\left|g_{k}(t)-g_{k+1}(t)\right|<p / 2$. Therefore

$$
\left|g_{k}\left(a_{k}\right)-g_{k+1}\left(a_{k}\right)\right|<p / 2 \text { and }\left|g_{k}\left(b_{k}\right)-g_{k+1}\left(b_{k}\right)\right|<p / 2 .
$$

Since

and

$$
\begin{aligned}
g_{k+1}\left(a_{k+1}\right) & <5 a_{k+1} / 4-a_{k+1} / 2+1 \\
& <3 a_{k} / 4+a_{k} / 2-1<g_{k}\left(a_{k}\right) \\
g_{k}\left(b_{k}\right) & <5 b_{k} / 4-a_{k} / 2+1 \\
& <3 b_{k+1} / 4+a_{k+1} / 2-1<g_{k+1}\left(b_{k+1}\right),
\end{aligned}
$$


there exist monotone increasing continuous functions

$$
\begin{array}{ll} 
& f_{k}^{-}:\left[a_{k+1}, a_{k}\right] \rightarrow \mathbf{R} \text { and } f_{k}^{+}:\left[b_{k}, b_{k+1}\right] \rightarrow \mathbf{R} \\
\text { satisfying } & f_{k}^{-}\left(a_{k+1}\right)=g_{k+1}\left(a_{k+1}\right), \quad f_{k}^{-}\left(a_{k}\right)=g_{k}\left(a_{k}\right), \\
& f_{k}^{+}\left(b_{k}\right)=g_{k}\left(b_{k}\right), \quad f_{k}^{+}\left(b_{k+1}\right)=g_{k+1}\left(b_{k+1}\right), \\
& \left|f_{k}^{-}(t)-g_{k+1}(t)\right|<p / 2, \quad a_{k+1} \leqslant t \leqslant a_{k}, \\
& \left|f_{k}^{+}(t)-g_{k+1}(t)\right|<p / 2, \quad b_{k} \leqslant t \leqslant b_{k+1} .
\end{array}
$$

Now, if we define $f: \mathbf{R} \rightarrow \mathbf{R}$ by

$$
f=g_{1} \cup\left(\bigcup_{k=1}^{\infty}\left(f_{k}^{-} \cup f_{k}^{+}\right)\right)
$$

then $f(0)=0$ and it is monotone increasing continuous. For any $t \in \mathbf{R}$, there is an $i>$ $k+1$ such that $d\left(y_{i} f(t), x f(t)\right)<a / 3$ whenever $a_{k+1} \leqslant t \leqslant a_{k}$ since $y_{k} f(t) \rightarrow z f(t)$. Note that

$$
\begin{aligned}
\left|f(t)-g_{i}(t)\right| & =\left|f_{k}^{-}(t)-g_{i}(t)\right| \\
& \leqslant\left|f_{k}^{-}(t)-g_{k+1}(t)\right|+\left|g_{k+1}(t)-g_{i}(t)\right| \\
& <p .
\end{aligned}
$$

Therefore

$$
\begin{aligned}
d\left(x f(t), x_{0} * t\right) \leqslant & d\left(x f(t), y_{i} f(t)\right)+d\left(y_{i} f(t), y_{i} g_{i}(t)\right) \\
& +d\left(y_{i} g_{i}(t), x_{0} * t\right) \\
& <a .
\end{aligned}
$$

The case $b_{k} \leqslant t \leqslant b_{k+1}, d\left(x f(t), x_{0} * t\right)<a$ follows in the same manner. It completes the proof.

\section{REFERENCES}

[1] R. Bowen and P. Walters, 'Expansive one-parameter flows', J. Differential Equations 12 (1972), 180-193.

[2] M. Hurley, 'Consequences of topological stability', J. Differential Equations 54 (1984), 60-72.

[3] J. Ombach, 'Consequences of the pseudo orbits tracing property and expansiveness', $J$. Austral. Math. Soc. 43 (1987), 301-313.

[4] M. Shub, Global stability of dynamical systems (Springer-Verlag, Berlin, Heidelberg, New York, 1987).

[5] R.F. Thomas, 'Stability properties of one-parameter flows', Proc. London Math. Soc. 45 (1982), 479-505. 
Department of Mathematics

Chungnam National University

Taejon, 305-764

Korea 